\title{
IBD sharing around the PPARG locus is not increased in dizygotic twins or their mothers
}

Busjahn et al ${ }^{1}$ reported finding linkage between PPARG (3p25) and being a dizygotic (DZ) twin. We differentiate, as do the authors, between the conception of $\mathrm{DZ}$ twins, and being the viable result of such a conception.

We observe no evidence of linkage to the region around the PPARG locus in

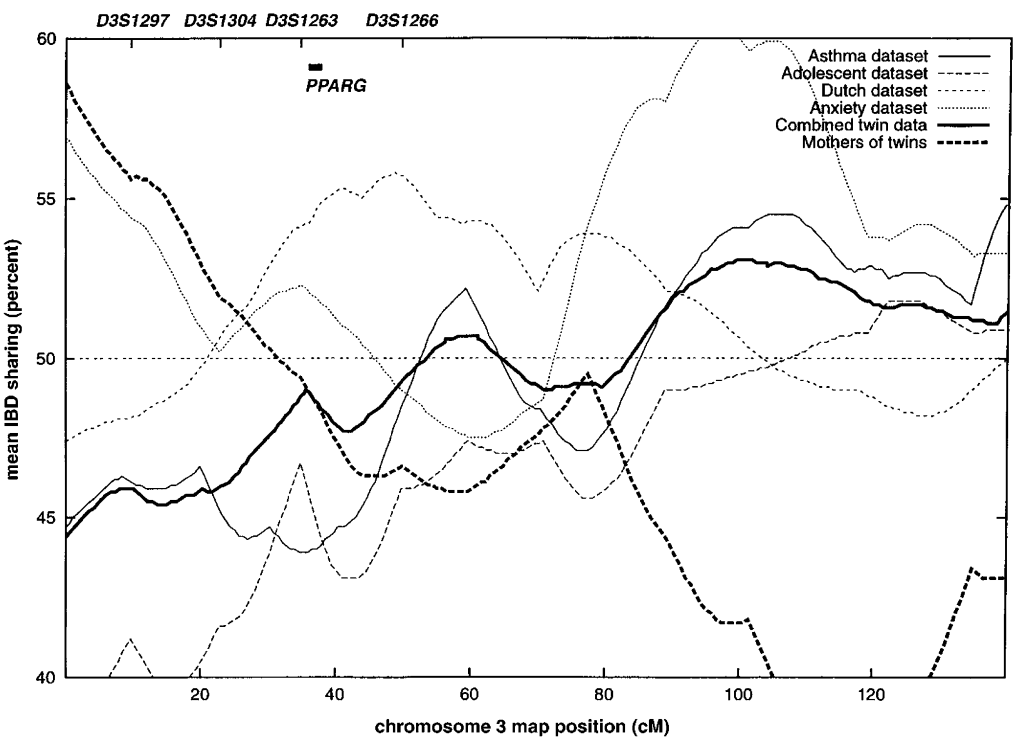

Fig. 1 Mean IBD sharing from five studies of DZtwins and twinning. similar result with 232 pairs of Australian adolescent DZ twins taking part in a longitudinal study of naevus development ${ }^{3}$ (0.444, 0.390-0.499), and a set of 125 Australian adult DZ twin pairs assessed for anxiety $^{4}(0.508,0.435-0.580)$. A Dutch scan of $160 \mathrm{DZ}$ twin pairs ${ }^{5}$ obtained slightly more encouraging results $(0.553$, 0.482-0.587, peak maximum lod score $(\mathrm{MLS})=0.57)$. Pooling all these samples gives $0.477(0.454-0.512)$ at the position of PPARG. The test for heterogeneity of sharing between studies was not significant $(P=0.10)$. In the combined dataset, the peak IBD sharing (MLS=0.70) is 50 cM closer to the centromere than PPARG.

Finally, in a sample of 203 Australian and New Zealand sister pairs where each had given birth to DZ twins ${ }^{6}$, sharing across the region is also not increased (0.433). We do not replicate linkage in the populations we study to survival of a twin pregnancy or polyovulation.

David Duffy ${ }^{1}$, Grant Montgomery ${ }^{1}$, Susan Treloar ${ }^{1}$, Andrew Birley ${ }^{1}$, Katherine Kirk ${ }^{1}$, Dorret Boomsma², Leo Beem², Eco de Geus $^{2}$, Eline Slagboom ${ }^{3}$, Joharna Knighton ${ }^{4}$, Peter Reed ${ }^{4}$ \& Nicholas Martin ${ }^{1}$ ${ }^{1}$ The Queensland Institute of Medical Research, Brisbane, Australia. 2Free University,

Amsterdam, The Netherlands. ${ }^{3}$ Gaubius Laboratory TNO/PG, Leiden, The Netherlands. ${ }^{4}$ Gemini Genomics, Cambridge, UK.

Received 4 January; accepted 27 June 2001

1. Busjahn, A. et al. Nature Genet. 26, 398-399 (2000). Duffy, D. et al. Am. Rev. Respir. Crit. Care Med. 157, 840-845 (1998).

3. Zhu, G. et al. Am. J. Hum. Genet. 65, 483-492 (1999).

4. Boomsmsa, D. et al. Twin Res. 3, 323-334 (2000).

5. Kirk, K. et al. Twin Res. 3, 299-309 (2000).

6. Montgomery, G. et al. J. Clin. Endocrinol. Metab. 85, 3391-3395 (2000). 